\title{
MicroRNA-1 regulates the proliferation of vascular smooth muscle cells by targeting insulin-like growth factor 1
}

\author{
$\mathrm{KUN} \mathrm{LIU}^{1}$, ZHANG YING $^{2}, \mathrm{XIA} \mathrm{QI}^{3}, \mathrm{YING} \mathrm{SHI}^{4}$ and QIANG TANG $^{1}$ \\ ${ }^{1}$ Department of Vascular Medicine, Peking University Shougang Hospital, Beijing; \\ ${ }^{2}$ Department of Cardiology, the First Affiliated Hospital of Dalian Medical University; \\ ${ }^{3}$ Transfusion Medicine Section, Department of Clinical Laboratory of Dalian Medical University, \\ Dalian, Liaoning; ${ }^{4}$ Beijing Youan Hospital, Beijing, P.R. China
}

Received September 15, 2014; Accepted May 29, 2015

DOI: $10.3892 /$ ijmm.2015.2277

\begin{abstract}
The aim of this study was to investigate the role of microRNAs (miRNAs or miRs) in vascular smooth muscle cell (VSMC) proliferation and to elucidate the underlying molecular mechanisms. In a previous study, using microarray analysis, differentially expressed miRNAs were identified in primary VSMCs isolated from the medial layer of the thoracic aorta obtained from spontaneously hypertensive rats (SHRs) and Wistar Kyoto (WKY) rats. Among others, miR-1 was identified to be downregulated in VSMCs from SHRs. Thus, in the present study, we focused on miR-1, the downregulation of which was confirmed by RT-qPCR and western blot analysis in VSMCs isolated from SHRs. We identified insulin-like growth factor 1 (IGF1) as a potential target gene of miR-1, and we subsequently validated IGF1 as a target gene of miR-1 by luciferase assay. The results revealed that the exogenous overexpression of miR-1 significantly suppressed the expression of IGF1. Additionally, we demonstrated that the downregulation of IGF1 by the introduction of miR-1 attenuated the proliferation of the VSMCs, suggesting that IGF1 is a target gene of miR-1 and that the effects of miR-1 are mediated through IGF1. In conclusion, the findings of our study demonstrate that miR-1 is significantly downregulated in VSMCs and that it is an important regulator of cell proliferation. Therefore, IGF1 may be involved in the regulation of VSMC proliferation by targeting miR-1.
\end{abstract}

\section{Introduction}

Hyperplasia of vascular smooth muscle cells (VSMCs) plays an essential role in the pathogenesis of a variety of cardiovascular diseases, such as hypertension and atherosclerosis (1). The deregulation of core genes palys a major role in the develop-

Correspondence to: Professor Qiang Tang, Department of Vascular Medicine, Peking University Shougang Hospital, 9 Jinyuan Zhuang Road, Shijingshan, Bejing 100144, P.R. China

E-mail: tangq_peking@126.com

Key words: microRNA-1, insulin-like growth factor 1, hypertension, vascular smooth muscle cells, proliferation ment of a wide spectrum of cardiovascular diseases, such as hypertension, heart failure and arteriosclerosis that are characterized by abnormally proliferating VSMCs (2-4). In the case of hypertension, it is generally accepted that the abnormally accelerated proliferation and enhanced contractile ability of VSMCs, significantly contribute to the pathophysiology of the disease (2,5). Insulin-like growth factor 1 (IGF1) is known to be a potent stimulator of VSMC proliferation (6). The expression levels of IGF1 have been found to be substantially upregulated in blood vessels following injury and in the aorta of hypertensive animals (7), and the ability of IGF1 to enhance VSMC proliferation requires the ligand occupancy of $\alpha_{\mathrm{V}} \beta_{3}$ integrin (8). However, the molecular mechanisms underlying the upregulation of IGF1 remain largely unknown.

MicroRNAs (miRNAs or miRs) are highly conserved endogenous small non-coding RNAs (approximately 22-25 nucleotides in length), and they are believed to be able to regulate the expression of up to one-third of all protein-coding genes in humans at the post-transcriptional level by binding to the 3' untranslated region (3'UTR) of the target genes, inhibiting the translation of mRNAs or promoting the degradation of mRNAs (9). miRNAs have been reported to play a role in various physiological and pathological processes, such as metabolism, cell differentiation, development, apoptosis and proliferation, as well as in oncogenesis (10). Accumulating evidence indicates that several specific miRNAs play a role in the regulation of VSMC proliferation, apoptosis and differentiation by targeting the post-transcriptional expression of multiple genes (11-14). For example, miR-145 has been found to be a regulator of VSMC phenotypic modulation and VSMC proliferation by targeting Krüppel-like factor 5 (KLF5) (12). Additionally, it has been reported that p27 (Kip1) and p57 (Kip2) are target genes of miR-221 and miR-222 and that they are responsible for their regulatory effects on VSMC proliferation and neointimal hyperplasia (14). These findings may lead to the identification of promising novel therapeutic targets for the treatment of a variety of proliferative vascular diseases, including hypertension and atherosclerosis.

It has been observed that spontaneously hypertensive rats (SHRs) and Wistar Kyoto (WKY) rats genetically share a common lineage, but markedly differ in their vascular phenotype (15), and it has also been reported that the proliferative activity of VSMCs 
isolated from SHRs (SHR-VSMCs) is markedly greater than that of VSMCs isolated from WKY rats (WKY-VSMCs) (16). In addition, in a previous study, Yu et al (17) identified differentially expressed miRNAs in SHR-VSMCs and WKY-VSMCs using microarray analysis. Thus, in this study, we performed reverse transcription-quantitative PCR (RT-qPCR) to verify the changes in the expression of the miRNAs identified by Yu et al (17) to be downregulated in SHR-VSMCs; these miRNAs include miR-1, miR-98, miR-34b and miR-500. We confirmed that miR-1 was significantly downregulated in the SHR-VSMCs compared with the controls. Using in silico analysis based on online predicting tools, such as TargetScan and miRDB, we identified IGF1 as a potential target gene of miR-1. Our hypothesis was that miR-1 may play a role in regulating the proliferation of VSMCs by targeting IGF1. To confirm this hypothesis, we validated that IGF1 is a target gene of miR-1 by luciferase assay and demonstrated that the exogenous overexpression of miR-1 significantly attenuated the proliferation of VSMCs.

\section{Materials and methods}

Cell culture. Rat aortic smooth muscle cells SMCs were isolated from the medial layer of the thoracic aorta of female SHRs and WKY rats (10 weeks old) and cultured in Dulbecco's modified Eagle's medium (DMEM; Lonza, Walkersville, MD, USA) supplemented with 10\% fetal bovine serum (FBS), penicillin and streptomycin (HyClone, Logan, UT, USA). The cells of passage 4-6 were employed in the experiment. The experimental protocol was approved by the Ethics Committee of Shougang Hospital, Peking University, Beijing, China. Human aortic SMCs (HASMCs) were cultured in SmGM-2 growth medium (both from Lonza) supplemented with 5\% FBS (HyClone) following the manufacturer's instructions.

Patient demographics and characteristics. Six patients who underwent open aortic arch reconstruction that required aortic dissection at Shougang Hospital were recruited in this study. Their demographic and clinical data are presented in Table I. The study protocol was approved by the Medical Ethics Committee of Shougang Hospital. Informed consent was obtained from all patients prior to enrollment. The SMCs were isolated as previously described (18). Briefly, resected vascular tissue was placed in DMEM with penicillin/streptomycin $(5 / 500 \mathrm{ml})$ and transferred to a super-clean bench. The vascular tissue was rinsed 3 times with phosphate-buffered saline (PBS) and the intima was removed. The tunica media was finely cut into 2-3-mm-thick sections in another $100-\mathrm{mm}$ culture dish, and $5 \mathrm{ml}$ of $0.1 \%$ type I collagenase (Invitrogen, Carlsbad, CA, USA) was added to the culture dish. Subsequently, the dish was placed in an incubator for $2 \mathrm{~h}$ at $37^{\circ} \mathrm{C}$. Digestion media were collected and filtrated using a cell strainer to remove the undigested explants and then centrifuged $\left(1,000 \mathrm{rpm}, 5 \mathrm{~min}, 4^{\circ} \mathrm{C}\right)$. The aforementioned procedures were repeated 3 times in order to harvest more cells.

Isolation of RNA and RT-qPCR. Total RNA was isolated using the miRNeasy Mini kit (Qiagen, Valencia, CA, USA) following the manufacturer's instructions, and the quality of the isolated RNA was evaluated using a NanoDrop spectrophotometer (Thermo Fisher Scientific, Waltham, MA, USA) and agarose electrophoresis. The PrimerScript RT reagent kit
Table I. Demographic data and clinical characteristics of the patients recruited in this study.

\begin{tabular}{lcccc}
\hline Patient ID & $\begin{array}{c}\text { Age } \\
\text { (years) }\end{array}$ & Gender & $\begin{array}{c}\text { Hypertension } \\
(\mathrm{mmHg})\end{array}$ & Diabetes \\
\hline 1 & 39 & $\mathrm{M}$ & $168 / 98$ & + \\
2 & 56 & $\mathrm{~F}$ & $173 / 105$ & + \\
3 & 63 & $\mathrm{M}$ & $182 / 112$ & + \\
4 & 58 & $\mathrm{M}$ & $118 / 75$ & - \\
5 & 42 & $\mathrm{~F}$ & $119 / 73$ & + \\
6 & 65 & $\mathrm{M}$ & $116 / 78$ & - \\
\hline
\end{tabular}

Table II. Primer sets used for qPCR, subcloning and mutagenesis of IGF1.

\begin{tabular}{ll}
\hline Name & \multicolumn{1}{c}{ Sequences } \\
\hline qPCR & \\
miRNA-1 & 5'-CTCAACTGGTGTCGTGGAGTCGGC \\
& AATTCAGTTGAGATACACAC-3' \\
& 5'-ACACTCCAGCTGGGTGGAATGTAA \\
& AGAAGT-3' \\
miRNA-34b & 5'-GTGCTCGGTTTGTAGGCAGT-3' \\
& 5'-GTGCCTTGTTTGATGGCAG-3' \\
miRNA-500 & 5'-CTGGCCGCCGATATTCACC-3' \\
& 5'-GGCAATCAAGTCAGCAAAATACC-3' \\
miRNA-98 & 5'-TGAGGTAGTAAGTTGTATTGTT-3' \\
& 5'-AGCGCAGATCAAAAGGAGACA-3' \\
IGF1 & 5'-AAATCAGCAGTCTTCCAAC-3' \\
& 5'-CTTCTGGGTCTTGGGCATGT-3' \\
U6 & 5'-CTCGCTTCGGCAGCACA-3' \\
& 5'-AACGCTTCACGAATTTGCGT-3' \\
Subcloning IGF1 3'UTR \\
Forward & 5'-GAAGACCCTCCTGAGGAGTG-3' \\
Reverse & 5'-GAACTAATTAATCAAACATG-3' \\
Mutagenesis of IGF1 \\
Forward & 5'-GAAATACACAAGTAATGTAAGGTA \\
& CATTGTCTTTAGGAGT-3' \\
Reverse & 5'-ACTCCTAAAGACAATGTACCTTACA \\
& TTACTTGTGTATTTC-3' \\
\hline
\end{tabular}

IGF1, insulin-like growth factor 1; 3'UTR, 3' untranslated region.

(Takara, Dalian, China) was used to synthesize the cDNA. The SYBR-Green real-time detection system was purchased from Bio-Rad Co., Ltd. (Hercules, CA, USA). U6 was used as an internal control to normalize the miR-1 and IGF1 mRNA expression levels. All fold changes were calculated using the $\Delta \Delta \mathrm{Ct}$ method. The primer sets used for amplification are listed in Table II. 
Overexpression of target miRNA. miR-1 mimics or anti-IGF1 siRNA (Ambion, Austin, TX, USA) were used in the experiments, and Lipofectamin 2000 (Invitrogen) was employed for the transfection of the HASMCs. Fluorescently labeled control oligo was first transfected and the transfection efficiency was evaluated by visual fluorescence microscopic analysis and flow cytometry. When the transfection efficiency was $>85 \%$, the transfection was considered successful. The in vitro experiments described below included cohorts transfected with 30 and 100 pmol of miR-1 mimics and 100 pmol of anti-IGF1 siRNA or the fluorescently labeled scrambled control oligo (negative control) known to have no effect on any human miRNA in order to minimize the non-specific effect.

MicroRNA target prediction. We searched two major online microRNA databases: TargetScan (www.targetscan.org) and miRDB (www.mirdb.org) to identify the candidate target genes of miR-1. Among all the candidate genes, we further narrowed down a short list of candidates based on the phyiopathological function of the genes.

Transwell invasion and migration assay. Transwell invasion assay was carried out in 24-well fitted inserts with membranes ( $8 \mathrm{~mm}$ pore size; Costar, Cambridge, MA, USA). As previously described (20), cell invasion was examined using a polycarbonate membrane cell culture insert (Costar, Corning Inc., Corning, NY, USA) coated with growth factor-reduced Matrigel (BD Biosciences, Bedford, MA, USA). The cells transfected with the control, miR-1 mimics and anti-IGF1 siRNA were treated with $10 \mathrm{mg} / \mathrm{ml}$ mitomycin C (Sigma-Aldrich, St. Louis, MO, USA) for $2 \mathrm{~h}$ and placed into the top of the wells at a density of $2.5 \times 10^{5}$ cells/well.

Cell survival assays. A modified 3-[4,5-dimethylthiazol-2-yl]2,5-diphenyltetrazolium bromide (MTT) assay was performed to examine HASMC viability. The HASMCs transfected with miR-1 mimics or anti-IGF1 siRNA were cultured in 96-well plates for $24 \mathrm{~h}$ and $10 \mu \mathrm{l}$ of MTT AB solution (Millipore, Billerica, MA, USA) were then added, followed by incubation for $4 \mathrm{~h}$. The formazan product was dissolved by the addition of $100 \mu \mathrm{l}$ of acidic isopropanol, and the absorbance was determined at $570 \mathrm{~nm}$ (reference wavelength $630 \mathrm{~nm}$ ) using an xMark Microplate Absorbance Spectrophotometer (Bio-Rad Co., Ltd.).

Apoptosis assays. Programmed cell death rates were assessed using a commercially available apoptosis assay (Sigma-Aldrich). The HASMCs were transfected with miR-1 mimics or antiIGF1 siRNA, and $1 \times 10^{5}$ cells were harvested and stained with $10 \mu \mathrm{l}$ FITC Annexin V and $10 \mu 1$ propidium iodide and sorted by FACS within $1 \mathrm{~h}$ (BD Biosciences, San Jose, CA, USA).

Western blot analysis. The transfected HASMCs were harvested and lysed, and the cell lysates were subjected to $10 \%$ PAGE. The separated proteins were transferred onto PVDF membranes blocked with TBST (10 mM Tris-Cl pH 8.0, $150 \mathrm{mM} \mathrm{NaCl}$, and $0.05 \%$ Tween-20) containing 5\% non-fat dry milk powder at room temperature for $1 \mathrm{~h}$. Subsequently, the membranes were incubated with primary antibodies directed against human IGF1 $(1: 2,000)$ and $\beta$-actin $(1: 1,500)$, and an HRP-conjugated goat-anti-rabbit secondary antibody $(1: 1,000)$

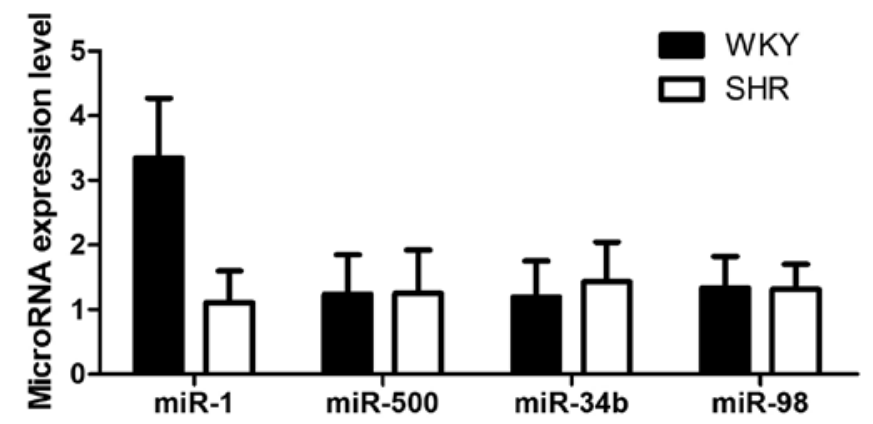

Figure 1. qPCR was used to evaluate and compare the expression levels of miR-1, miR-500, miR-34b and miR-98 in the WKY-VSMCs and SHR-VSMCs. VSMCs, vascular smooth muscle cells; WKY, denotes cells from Wistar Kyoto rats; SHR, denotes cells from spontaneously hypertensive rats.

(both from Cell Signaling Technology, Danvers, MA, USA). Exposed films were scanned and fluorescence signals were detected using an ECL kit (Pierce, Rockford, IL, USA).

Luciferase assay. The PCR product was then cloned into the pRL-SV40 vector (Promega, Fitchburg, WI, USA) to replace the 3'UTR of Renilla luciferase, and the QuickChange XL sitedirected mutagenesis kit (Stratagene, La Jolla, CA, USA) was used to introduce the variant. Luciferase assay was conducted on the HASMCs, and the cells were seeded at $1 \times 10^{5}$ cells/well in 24-well plates. Twelve hours later, the cells were transfected using Lipofectamin 2000 (Invitrogen) according to the manufacturer's instructions. In each well, $500 \mathrm{ng}$ of the wild-type or mutant construct and $50 \mathrm{ng}$ of the control vector (control oligo) were co-transfected with $100 \mathrm{pmol}$ miR-1 mimics or the negative control (Ambion). Twenty-four hours after transfection, the cells were harvested by the addition of passive lysis buffer (Promega). Luciferase activity in the cell lysates was determined using the Dual Luciferase assay system (Promega) with a TD-20/20 luminometer (Turner Biosystems, Sunnyvale, CA, USA).

Statistical analysis. All data are presented as the means \pm SD. One-way ANOVA was used for comparisons between samples ( $>2$ groups), and an independent t-test was used for comparisons between 2 groups. A value of $\mathrm{P}<0.05$ was considered to indicate a statistically significant difference. All experiments were performed at least 3 times. Statistical analysis was performed using the SPSS software package (version 19; SPSS, Inc., Chicago, IL, USA).

\section{Results}

Comparison of miRNA expression profiles between $S H R$-VSMCs and WKY-VSMCs. In the present study, we focused on miR-1, miR-500, miR-34b and miR-98, as these miRNAs have been previously reported to be significantly downregulated in SHR-VSMCs compared with WKY-VSMCs, based on the results of microarray-based miRNA expression profiling analysis (17). RT-qPCR was performed using the primary VSMCs isolated from the medial layer of the thoracic aorta obtained from SHRs and WKY rats, and we observed a 3.29-fold decrease in the expression levels of miR-1 in the SHR-VSMCs compared with the WKY-VSMCs $(\mathrm{P}<0.01$; Fig. 1), whereas 
A

Hsa-miR-1 targets IGF1

IGF1 WT 3’ UTR (151-157)

Hsa-miR-1

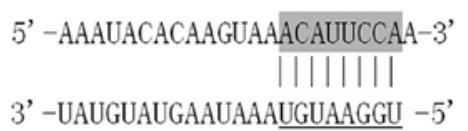

IGF1 Mut 3' UTR (151-157) 5’-AAAUACACAAGUAAUGUAAGGUA-3’
B

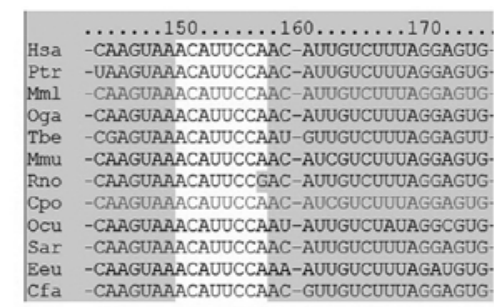

$\mathrm{C}$

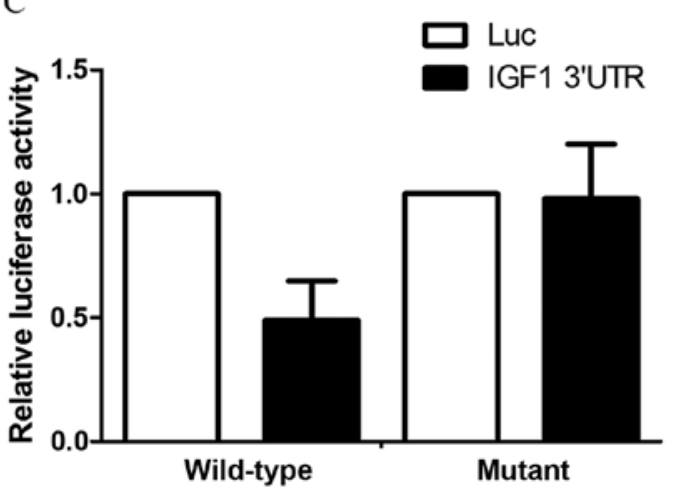

Figure 2. (A) Schematic introduction and comparison of miR-1, wild-type IGF1 3'UTR and mutant IGF1 3'UTR. (B) The 'seed sequence' is highly conserved among species. (C) comparison of the relative luciferase activity between wild-type and mutant IGF1 3'UTR. IGF1, insulin-like growth factor 1; 3'UTR, $3^{\prime}$ untranslated region. Luc, background fluorescence (by transfection with Renilla).

the expression levels of the miRNAs were similar between the 2 groups. Thus, miR-1 was further investigated to determine its potential biological function.

Validation of IGF1 as a target gene of miR-1 using the luciferase reporter system. Using in silico analysis based on online predicting tools, such as TargetScan and miRDB, we identified IGF1 as a potential target gene of miR-1 (Fig. 2A), and the 'seed sequence' in the 3'UTR of IGF1 was found to be highly conserved among species (Fig. 2B), suggesting that such a 'seed sequence' may have an important function. To validate the target gene, we subcloned the 3'UTR of IGF1 into the pRL-SV40 vector to replace the 3'UTR of Renilla luciferase, and performed luciferase assay to compare the inhibitory effects between the wild-type 3'UTR and the mutant as described in the Materials and methods. The results revealed that co-transfection with the miR-1 mimic significantly suppressed luciferase activity when transfected together with the wild-type 3'UTR of IGF1, but not the mutant one (Fig. 2C).

Inhibition of IGF1 expression in VSMCs by transfection with miR-1 mimics. Additionally, the HASMCs were transfected with miR-1 mimics (30 and 100 pmol) and IGF1-specific siRNA, and the IGF1 protein levels were quantified by western blot analysis. Fig. 3E shows the step-wise downregulation of IGF1 expression in the HASMCs transfected with an increasing amount of miR-1 mimics (30 and 100 pmol). The silencing effect of transfection with 100 pmol of the miR-1 mimics was comparable with that of transfection with 100 pmol of the antiIGF1 siRNA.

Effects of the overexpression of miR-1 on VSMC proliferation. The HAMSCs were transfected with the miR-1 mimics (30 and $100 \mathrm{pmol}$ ) and anti-IGF1 siRNA prior to the proliferation assay. MTT assay was used to measure cell proliferation. The results revealed that the exogenous overexpression of miR-1 significantly inhibited the proliferation of the HAMSCs (Fig. 3). After incubation for $48 \mathrm{~h}$, significant inhibitory effects on cell proliferation were observed in the cells transfected with the miR-1 mimis or with anti-IGF1 siRNA compared with those transfected with the negative control. In the HASMCs, the inhibitory rates were 75\% (anti-IGF1 siRNA), 22\% (30 pmol miR-1 mimics) and 80\% (100 pmol miR-1 mimics) following incubation for $48 \mathrm{~h}$ (Fig. 3F). This suggested that the overexpression of miR-1 inhibited the proliferation of the VSMCs in vitro. Furthermore, we performed Transwell invasion assay with the cells being treated with mitomycin $\mathrm{C}$ that was used to eliminate the possible effect of cell growth on cell invasion. The results revealed that the inhibition of miR-1 in the HASMCs did not affect cell invasiveness (data not shown).

Comparison of IGF1 expression between the SHR-VSMCs and WKY-VSMCs. We examined the SHR-VSMCs and WKY-VSMCs to determine the expression patterns of IGF1 in these cells. The IGF1 mRNA expression levels were measured by RT-qPCR, and we found that the mRNA levels of IGF1 in the WKY-VSMCs were significantly lower than those in the SHR-VSMCs (Fig. 4B). The IGF1 protein levels were also measured using western blot anlaysis. The results revealed that the protein expression levels of IGF1 in the SHR-VSMCs were substantitally higher than those in the WKY-VSMCs (Fig. 4A).

Comparison of IGF1 expression levels between VSMCs collected from patients with severe hypertension and the normal blood pressure control group. VSMCs were isolated 
A

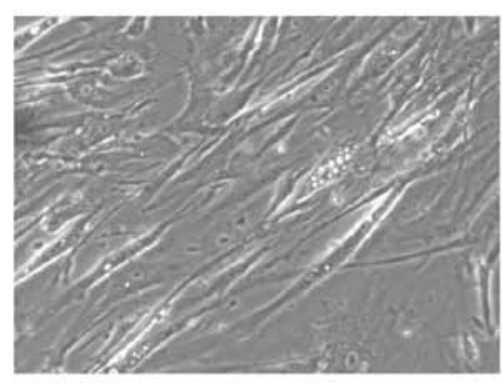

B

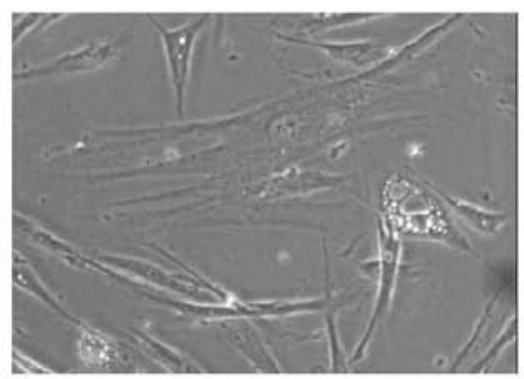

C

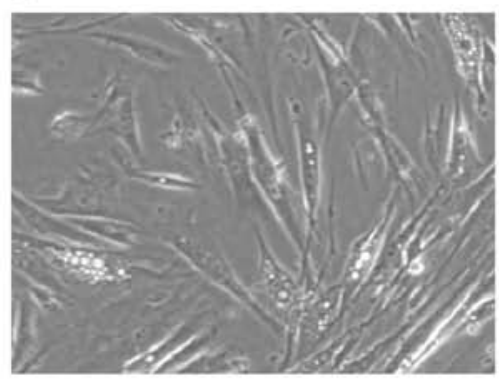

D

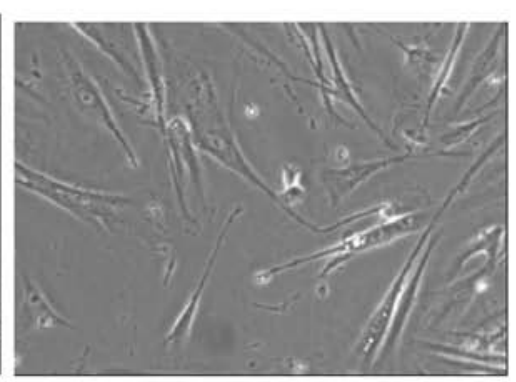

E
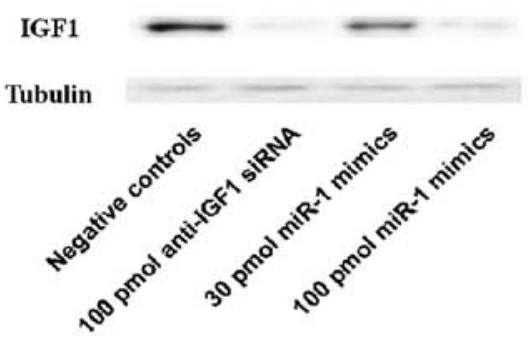

F

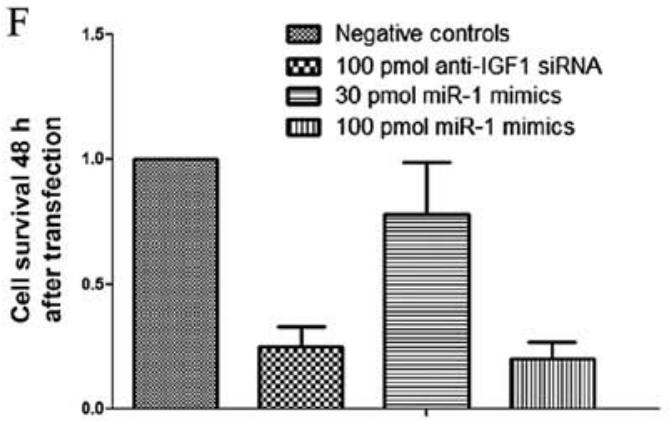

Figure 3. (A) Microscopic HASMC proliferation status $48 \mathrm{~h}$ after transfection with negative controls. (B) Microscopic HASMC proliferation status $48 \mathrm{~h}$ after transfection with 100 pmol anti-IGF1 siRNA. (C) Microscopic HASMC proliferation status $48 \mathrm{~h}$ after transfection with 30 pmol miR-1 mimics. (D) Microscopic HASMC proliferation status $48 \mathrm{~h}$ after transfection with 100 pmol miR-1 mimics. (E) Silencing effects of 100 pmol anti-IGF1 siRNA and $30 / 100$ pmol miR-1 mimics on the protein expression levels of IGF1. (F) Effects of 100 pmol anti-IGF1 siRNA and 30/100 pmol miR-1 mimics on the proliferation of VSMCs. HASMCs, human aortic smooth muscle cells; IGF1, insulin-like growth factor 1; VSMCs, vascular smooth muscle cells.

\section{A IGF1}

Tubulin

$$
\text { WKY SHR }
$$

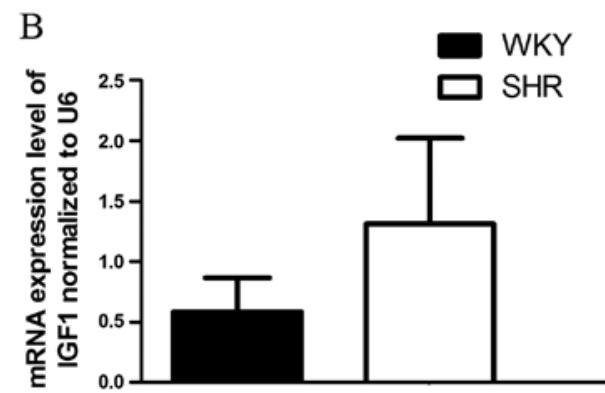

Figure 4. Comparison of (A) mRNA and (B) protein expression levels of IGF1 between WKY-VSMCs and SHR-VSMCs. IGF1, insulin-like growth factor 1; VSMCs, vascular smooth muscle cells; WKY, denotes cells from Wistar Kyoto rats; SHR, denotes cells from spontaneously hypertensive rats.

from the vascular tissue collected from 6 patients (hypertension, 3; normal blood pressure, 3) who had undergone open aortic arch reconstruction that required aortic dissection (HTN-VSMCs vs. normal-VSMCs). The mRNA and protein expression levels of IGF1 were compared between the 2 groups of cells by RT-qPCR and western blot analysis. We found that both the protein and mRNA expression levels of IGF1 were markedly higher in the HTN-VSMCs than in the normal-VSMCs (Fig. 5B and C, respectively). Similarly, the expression levels of miR-1 were significantly lower in the HTN-VSMCs than in the normal-VSMCs (Fig. 5A).

Increased expression of miR-1 induces the apoptosis of HASMCs. As IGF1 functions as an anti-apoptotic protein and the exogenous expression of miR-1 causes a reduction in the enzyme (21), we examined the effects of miR-1 on the apoptosis of HASMCs by flow cytometry. We found that transfection of the cells with miR-1 mimics and anti-IGF1 siRNA promoted the apoptosis of HASMCs (Fig. 6).

\section{Discussion}

The regulatory role of miRNAs in the control of VSMC proliferation and the phenotypic switch has been well described in the literature (12). Furthermore, it has been demonstrated that proliferating VSMCs significantly contribute to the pathogenesis of a wide spectrum of cardiovascular diseases, such as atherosclerosis and hypertension $(3,4)$. In the present study, we demonstrated that the expression of miR-1 was substantially downregulated in the SHR-VSMCs compared with the WKY-VSMCs, and that the exogenous overexpression of miR-1 markedly inhibited the proliferation of the VSMCs; these effects were mediated by the downregulation of its target gene, IGF1. Our results are consistent with the results of previous studies on the role of miR-1 in the growth and proliferation of cardiomyocytes and skeletal myoblasts $(19,20,22,23)$.

The results of the present study demonstrated that transfection of the cells with miR-1 inhibited VSMC proliferation; these results are consistent with the inhibitory role of miR-1 in the proliferation of other types of muscle cells, such as 

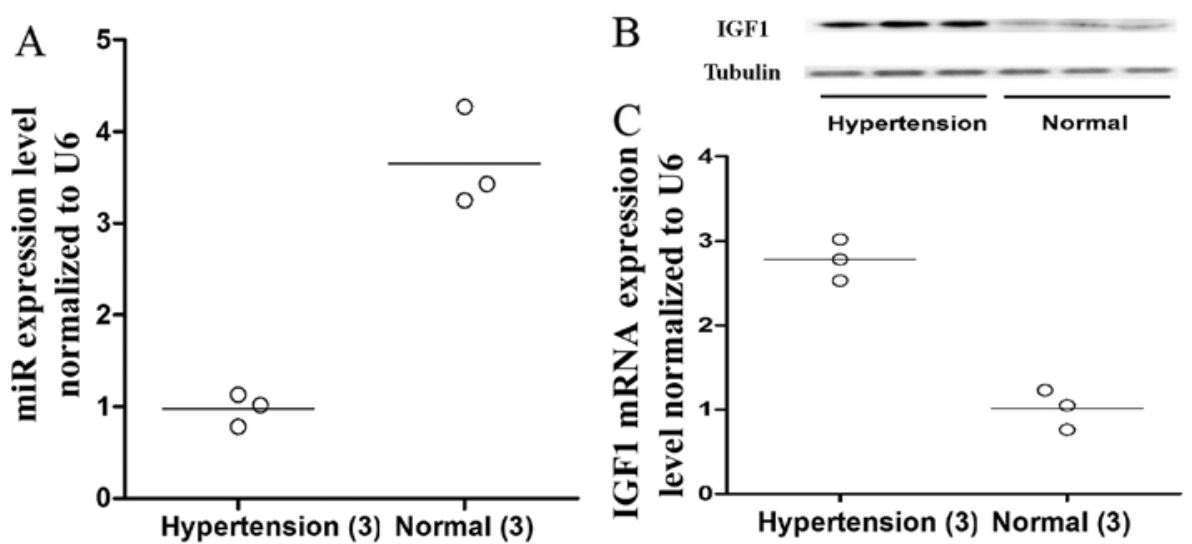

Figure 5. Comparison of (A) miR-1 expression and (B) IGF1 protein and (C) IGF1 mRNA expression levels in VSMCs obtained from patients who underwent aortic dissection with or without hypertension. IGF1, insulin-like growth factor 1; VSMCs, vascular smooth muscle cells.
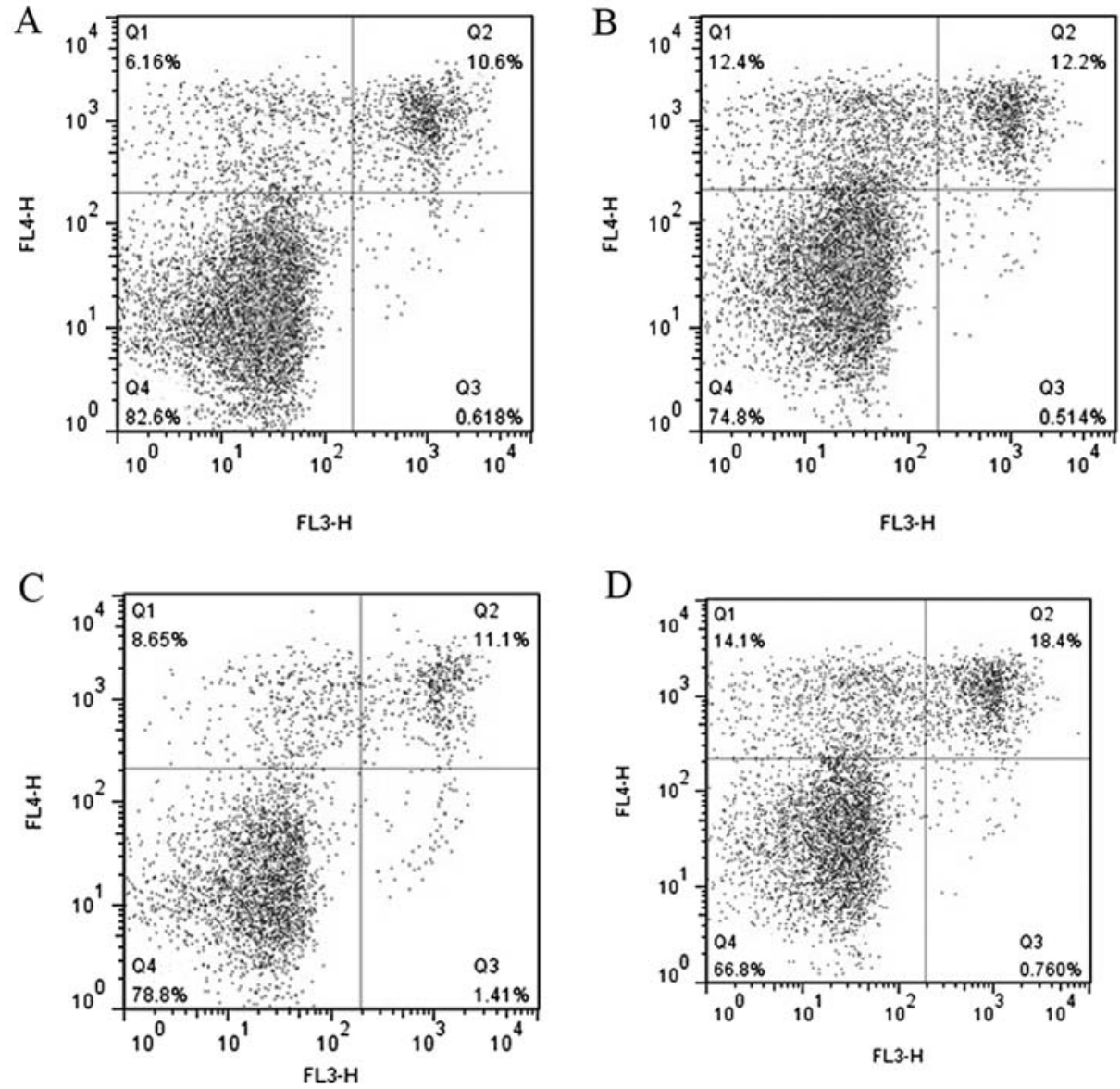

Figure 6. (A) Apoptosis of HASMCs transfected with negative controls. (B) Apoptosis of HASMCs transfected with 100 pmol of anti-IGF1 siRNA. (C) Apoptosis of HASMCs transfected with 30 pmol of miR-1 mimics. (D) Apoptosis of HASMCs transfected with 100 pmol of miR-1 mimics. HASMCs, human aortic smooth muscle cells; IGF1, insulin-like growth factor 1.

cardiomyocytes and skeletal myoblasts $(22,23)$. miR-1 has also been reported to play a similar role in regulating the growth of cancer cells (24). Our data suggest that the downregulation of miR- 1 contributes to a higher proliferation rate of VSMCs isolated from SHRs, providing a potential explanation for the lower proliferation rate of VSMCs obtained from WKY rats compared to those from SHRs. Furthermore, considering the inhibitory role of miR-1 in the control of the proliferation of
VSMCs, the deregulation of miR-1 signaling may also facilitate VSMC proliferation in some pathophysiological context, such as atherosclerosis and restenosis following angioplasty. A number of genes have been suggested to be potential targets of miR-1 in various types of cells and tissues, including BRG1, which is a subunit of the SWI/SNF complex (25), morphogenetic protein 2 (BMP2) (26), bone morphogenetic protein receptor type-1B (BMPR1B), transforming growth factor (TGF) $-\beta$ (27), 
transforming growth factor, $\beta$ receptor III (TGFBR3) and the downstream signaling mediators, SMAD2 and SMAD4 (TargetScan). Using in silico analysis based on online predicting tools, such as TargetScan and miRDB, we identified IGF1 as potential target gene of miR-1, and we subsequently validated IGF1 as a target gene of miR-1 by luciferase assay, and demonstrated that the exogenous overexpression of miR-1 significantly suppressed the expression of IGF1.

IGF1 is an ubiquitous factor which exerts pleiotropic effects on a variety of cell types and can activate the signaling pathway downstream of the IGF1 receptor (IGF1R) by binding to the receptor, which has been shown to play a crucial role in the control of cell proliferation, differentiation, transformation and survival (28). IGF1R-dependent signaling is crucial for the survival of many cell types, including VSMCs (28). In this study, we demonstrated that the downregulation of IGF1 by the introduction of miR-1 attenuated the proliferation of the VSMCs, and the inhibitory effect of miR-1 on cell proliferation was comparable to that observed with transfection with antiIGF1 siRNA, suggesting that IGF1 is a target gene of miR-1 and that the anti-proliferative effects of miR-1 are mediated through IGF1. It has been previously demonstrated that VSMCs derived from atherosclerotic plaques (pVSMCs) have a defect in IGF1-dependent survival signaling (29), and are more sensitive to apoptosis than cells derived from normal control vessels (30). Furthermore, it has been demonstrated that oxidative stress reduces IGF1R expression and induces VSMC apoptosis in culture (31-34). The downregulation of IGF1/IGF1R signaling has also been observed in atherosclerotic lesions, indicating that IGF1/IGF1R-dependent survival regulates apoptosis in vivo $(6,35)$. VSMCs lacking IGF1/IGF1R also represent an increased sensitivity to multiple pro-apoptotic stimuli, such as tumor suppressor genes and death receptor ligation $(36,37)$, suggesting that the defective IGF1/IGF1R signaling pathway alone is an important cause of the reduced survival of VSMCs. In this study, we found that the inhibitory effect of miR-1 on the proliferation of VSMCs was mediated by its ability to induce apoptosis by suppressing the expression of IGF1. A major downstream effector of IGF1R signaling is the serine/threonine kinase, Akt [also known as protein kinase B (PKB)]. Akt phosphorylates a variety of targets involved in glucose metabolism and cell differentiation, proliferation and survival (38). It has been shown that VSMCs in plaque also exhibit a reduced activation of Akt in response to IGF1 treatment (29), suggesting that Akt mediates IGF1R-dependent signaling in these cells. It has also been demonstrated that the activation of Akt is necessary and/or sufficient for VSMC survival in response to apoptotic stimuli, and that Akt-dependent phosphorylation and the subsequent inactivation of forkhead box O3a (FOXO3a) and glycogen synthase kinase-3 (GSK3) is important for VSMC survival (28).

To further establish the role of IGF1 in mediating the effects of miR-1 in VSMCs, we evaluated and compared the effects of miR-1 mimics and anti-IGF1 siRNA on the expression of IGF1 and on the proliferation status of VSMCs. As shown in Fig. 3, transfection with 100 pmol of miR-1 mimics and 100 pmol of anti-IGF1 siRNA had a similar effect on the knockdown the protein expression levels of IGF1, whereas the inhibitory effects of miR-1 mimics on cell proliferation were more prominent than those of anti-IGF1 siRNA. We reasoned that the inhibitory effects of miR-1 on VSMC proliferation were mediated, at least in part, through some other mediators, such as Pim-1, a gene encoding an oncogenic serine/threonine kinase, which is required for injury-induced neointima formation and VSMC proliferation (39).

Additionally, miR-1 has been reported to be involved in regulating the contractile phenotype of VSMCs in a myocardindependent manner which has important physiological relevance to the control of blood pressure in vivo (40). Phenotypic plasticity is an important characteristic of mature VSMCs. Different phenotypes of VSMCs exist in the normal blood vessel wall and the dysregulation of the phenotype differentiation contributes to the development of hypertension (41-43).

In conclusion, in the present study, we demonstrate that the downregulation of miR-1 inhibits VSMC proliferation in SHR-VSMCs, at least in part through the downregulation of IGF1. It is crucial to determine whether the miR-1-IGF1 pathway serves as a therapeutic target in proliferative vascular diseases.

\section{Acknowledgements}

The study was fully sponsored by the National Natural Science Foundation of China (grant no. 81100288).

\section{References}

1. Yu X and Li Z: MicroRNAs regulate vascular smooth muscle cell functions in atherosclerosis (Review). Int J Mol Med 34: 923-933, 2014.

2. Bornfeldt KE and Krebs EG: Crosstalk between protein kinase A and growth factor receptor signaling pathways in arterial smooth muscle. Cell Signal 11: 465- 477, 1999.

3. Somjen D, Knoll E, Sharon O, Many A and Stern N: Calciotrophic hormones and hyperglycemia modulate vitamin $\mathrm{D}$ receptor and 25 hydroxyy vitamin D 1- $\alpha$ hydroxylase mRNA expression in human vascular smooth muscle cells. J Steroid Biochem Mol Biol 148: 210-213, 2015.

4. Liu N, Bezprozvannaya S, Williams AH, Qi X, Richardson JA, Bassel-Duby R and Olson EN: microRNA-133a regulates cardiomyocyte proliferation and suppresses smooth muscle gene expression in the heart. Genes Dev 22: 3242-3254, 2008.

5. Do e Z, Fukumoto Y, Takaki A, et al: Evidence for Rho-kinase activation in patients with pulmonary arterial hypertension. Circ J 73: 1731-1739, 2009.

6. Delafontaine P, Song YH and Li Y: Expression, regulation, and function of IGF-1, IGF-1R, and IGF-1 binding proteins in blood vessels. Arterioscler Thromb Vasc Biol 24: 435-444, 2004.

7. Fath KA, Alexander RW and Delafontaine P: Abdominal coarctation increases insulin-like growth factor I mRNA levels in rat aorta. Circ Res 72: 271-277, 1993.

8. Maile LA, Busby WH, Sitko K, et al: Insulin-like growth factor-I signaling in smooth muscle cells is regulated by ligand binding to the 177CYDMKTTC184 sequence of the beta3-subunit of alphaVbeta3. Mol Endocrinol 20: 405-413, 2006.

9. Valinezhad Orang A, Safaralizadeh R1 and KazemzadehBavili M: Mechanisms of miRNA-mediated gene regulation from common downregulation to mRNA-specific upregulation. Int J Genomics 2014: 970607, 2014.

10. Cho WC: OncomiRs: the discovery and progress of microRNAs in cancers. Mol Cancer 6: 60, 2007.

11. Chan MC, Hilyard AC, Wu C, et al: Molecular basis for antagonism between PDGF and the TGFbeta family of signalling pathways by control of miR-24 expression. EMBO J 29: 559-573, 2010.

12. Elia L, Quintavalle M, Zhang J, et al: The knockout of miR-143 and -145 alters smooth muscle cell maintenance and vascular homeostasis in mice: correlates with human disease. Cell Death Differ 16: 1590-1598, 2009.

13. Zhang C: MicroRNA-145 in vascular smooth muscle cell biology: a new therapeutic target for vascular disease. Cell Cycle 8: 3469-3473, 2009. 
14. Liu X, Cheng Y, Zhang S, et al: A necessary role of miR-221 and miR-222 in vascular smooth muscle cell proliferation and neointimal hyperplasia. Circ Res 104: 476-487, 2009.

15. Louis WJ and Howes LG: Genealogy of the spontaneously hypertensive rat and Wistar-Kyoto rat strains: Implications for studies of inherited hypertension. J Cardiovasc Pharmacol 16 (Suppl 7): S1-S5, 1990.

16. Uehara Y, Numabe A, Kawabata Y, et al: Rapid smooth muscle cell growth and endogenous prostaglandin system in spontaneously hypertensive rats. Am J Hypertens 4: 806-814, 1991.

17. Yu ML, Wang JF, Wang GK, et al: Vascular smooth muscle cell proliferation is influenced by let-7d microRNA and its interaction with KRAS. Circ J 75: 703-709, 2011.

18. Lu S, Sun X, Hong T, et al: Isolation and culture of smooth muscle cells from human acute type A aortic dissection. J Cardiothorac Surg 8: 83, 2013.

19. Zhao Y, Ransom JF, Li A, et al: Dysregulation of cardiogenesis, cardiac conduction, and cell cycle in mice lacking miRNA-1-2. Cell 129: 303-317, 2007

20. van RooijE,Sutherland LB, QiX, et al: Control of stress-dependent cardiac growth and gene expression by a microRNA. Science 316 575-579, 2007.

21. Walenkamp MJ, Losekoot M and Wit JM: Molecular IGF-1 and IGF-1 receptor defects: from genetics to clinical management. Endocr Dev 24: 128-137, 2013.

22. Chen JF, Mandel EM, Thomson JM, et al: The role of microRNA-1 and microRNA-133 in skeletal muscle proliferation and differentiation. Nat Genet 38: 228-233, 2006.

23. Zhao Y, Samal E and Srivastava D: Serum response factor regulates a muscle-specific microRNA that targets Hand 2 during cardiogenesis. Nature 436: 214-220, 2005.

24. Nasser MW, Datta J, Nuovo G, et al: Down-regulation of micro-RNA-1 (miR-1) in lung cancer. Suppression of tumorigenic property of lung cancer cells and their sensitization to doxorubicin-induced apoptosis by miR-1. J Biol Chem 283 33394-33405, 2008.

25. Rodriguez-Nieto S and Sanchez-Cespedes M: BRG1 and LKB1: tales of two tumor suppressor genes on chromosome 19p and lung cancer. Carcinogenesis 30: 547-554, 2009.

26. Hou X, Tang Z, Liu H, et al: Discovery of microRNAs associated with myogenesis by deep sequencing of serial developmental skeletal muscles in pigs. PLoS One 7: e52123, 2012.

27. Luong HT, Chaplin J, McRae AF, Medland SE, Willemsen G, Nyholt DR, Henders AK, Hoekstra C, Duffy DL, et al: Variation in BMPR1B, TGFRB1 and BMPR2 and control of dizygotic twinning. Twin Res Hum Genet 14: 408-416, 2011.

28. Allard D, Figg N, Bennett MR and Littlewood TD: Akt regulates the survival of vascular smooth muscle cells via inhibition of FoxO3a and GSK3. J Biol Chem 283: 19739-19747, 2008.

29. Patel VA, Zhang QJ, Siddle K, et al: Defect in insulin-like growth factor-1 survival mechanism in atherosclerotic plaque-derived vascular smooth muscle cells is mediated by reduced surface binding and signaling. Circ Res 88: 895-902, 2011.
30. Bennett MR, Evan GI and Schwartz SM: Apoptosis of human vascular smooth muscle cells derived from normal vessels and coronary atherosclerotic plaques. J Clin Investig 95: 2266-2274, 1995.

31. Kavurma MM, Figg N, Bennett MR, et al: Oxidative stress regulates IGF1R expression in vascular smooth-muscle cells via p53 and HDAC recruitment. Biochem J 407: 79-87, 2007.

32. Pedruzzi E, Guichard C, Ollivier V, et al: $\mathrm{NAD}(\mathrm{P}) \mathrm{H}$ oxidase Nox-4 mediates 7-ketocholesterol-induced endoplasmic reticulum stress and apoptosis in human aortic smooth muscle cells. Mol Cell Biol 24: 10703-10717, 2004.

33. Sandberg EM and Sayeski PP: Jak2 tyrosine kinase mediates oxidative stress-induced apoptosis in vascular smooth muscle cells. J Biol Chem 279: 34547-34552, 2004.

34. Song HJ, Lee TS, Jeong JH, Min YS, Shin CY and Sohn UD Hydrogen peroxide-induced extracellular signal-regulated kinase activation in cultured feline ileal smooth muscle cells. J Pharmacol Exp Ther 312: 391-398, 2005.

35. Okura Y, Brink M, Zahid AA, Anwar A and Delafontaine P: Decreased expression of insulin-like growth factor-1 and apoptosis of vascular smooth muscle cells in human atherosclerotic plaque. J Mol Cell Cardiol 33: 1777-1789, 2001.

36. Bennett MR, Littlewood TD, Schwartz SM and Weissberg PL: Increased sensitivity of human vascular smooth muscle cells from atherosclerotic plaques to p53-mediated apoptosis. Circ Res 81: 591-599, 1997.

37. Chan SW, Hegyi L, Scott S, et al: Sensitivity to Fas-mediated apoptosis is determined below receptor level in human vascular smooth muscle cells. Circ Res 86: 1038-1046, 2000.

38. Liang Y, Jing Z, Deng H, Li Z, Zhuang Z, Wang S and Wang Y. Soluble epoxide hydrolase inhibition ameliorates proteinuriainduced epithelial-mesenchymal transition by regulating the PI3K-Akt-GSK-3 $\beta$ signaling pathway. Biochem Biophys Res Commun 463: 70-75, 2015.

39. Liu Y, Zhang J, Yi B, Chen M, Qi J, Yin Y, Lu X, Jasmin JF and Sun J: Nur77 suppresses pulmonary artery smooth muscle cell proliferation through inhibition of the STAT3/Pim-1/NFAT pathway. Am J Respir Cell Mol Biol 50: 379-388, 2014.

40. Hamfjord J, Stangeland AM, Hughes T, et al: Differential expression of miRNAs in colorectal cancer: comparison of paired tumor tissue and adjacentnormal mucosa using high-throughput sequencing. PLoS One 7: e34150, 2012.

41. Mitra S, Goyal T and Mehta JL: Oxidized LDL, LOX-1 and atherosclerosis. Cardiovasc Drugs Ther 25: 419-429, 2011.

42. Stoneman VE and Bennett MR: Role of apoptosis in atherosclerosis and its therapeutic implications. Clin Sci (Lond) 107: 343-354, 2004.

43. Conover CA, Bale LK, Harrington SC, et al: Cytokine stimulation of pregnancy-associated plasma protein A expression in human coronary artery smooth muscle cells: inhibition by resveratrol. Am J Physiol Cell Physiol 290: C183-C188, 2006. 